AIAA-2004-2219

2nd AIAA Flow Control Conference, June 28-July 1, 2004, Portland, Oregon

\title{
The Isolated Synthetic Jet in Crossflow: A Benchmark for Flow Control Simulation
}

\author{
Norman W. Schaeffler* and Luther N. Jenkins ${ }^{\dagger}$ \\ NASA Langley Research Center, Hampton, Virginia, United States of America
}

\begin{abstract}
An overview of the data acquisition, reduction, and uncertainty of experimental measurements of the flowfield created by the interaction of an isolated synthetic jet and a turbulent boundary layer is presented. The experimental measurements were undertaken to serve as the second of three computational fluid dynamics validation databases for Active Flow Control. The validation databases were presented at the NASA Langley Research Center Workshop on CFD Validation of Synthetic Jets and Turbulent Separation Control in March, 2004. Detailed measurements were made to document the boundary conditions for the flow and also for the phase-averaged flowfield itself. Three component Laser-Doppler Velocimetry, 2-D Particle Image Velocimetry, and Stereo Particle Image Velocimetry were utilized to document the phase averaged velocity field and the turbulent stresses.
\end{abstract}

\section{Introduction}

THE "active" in active flow control (AFC) implies that an actuator is attempting to exert control over a 1 flow in a time-dependent sense. By varying the actuator output, or turning it completely on and off, the flow can be tailored in different ways at different times. In fact, the output of the actuator itself is, more than likely, an unsteady flow, exploiting the additional dynamics of startup to maximize the vortical impulse that the actuator can deliver to the flow. Moreover, the underlying flow that we wish to control is very often an unsteady, or time-dependent, flow. Flows with separated regions, flows with noise-producing free shear layers, flows for which we wish to enhance mixing, all of these are dominated by structures that exist only in a time-dependent sense. The design of future AFC actuators and actuator configurations, for both laboratory use and actual flight vehicle use, would be greatly enhanced by the availability of computational tools capable of predicting the unsteady flows typical of an AFC configuration. Advances in computational fluid dynamics (CFD) over the last decade have indicated that such a tool is conceivable in the near future, but one of the principle impediments in its development is the lack of high-quality experiments to validate such a tool against. ${ }^{1}$ This is not to say that there are not high-quality experiments documented in the open literature, but to say that the type of details that need to be documented for the experiment to be useful as a validation experiment are not typically documented in the literature. This is especially true when it comes to boundary conditions.

To address this lack of validation data, specifically with respect to AFC, a set of three validation experiments were conducted at NASA Langley. Each of the three experiments were designed with close collaboration between the experimentalists and the CFD community to define the geometries used in the three cases, the actuator details, and the type and location of the data to be acquired. The three experiments form the validation databases for the NASA Langley Research Center Workshop on CFD Validation of Synthetic Jets and Turbulent Separation Control. The workshop was conducted in March, 2004, in association with the

\footnotetext{
* Research Scientist, Flow Physics and Control Branch, Member AIAA

${ }^{\dagger}$ Research Scientist, Flow Physics and Control Branch

This material is declared a work of the U.S. Government and is not subject to copyright protection in the United States.
} 
European Research Community On Flow, Turbulence And Combustion (ERCOFTAC), the U.S. Air Force Office of Scientific Research (AFOSR), the International Association of Hydraulic Engineering and Research (IAHR), QNET-CFD, and the National Institute of Aerospace (NIA). An overview of the workshop itself is presented in Rumsey, et. al. ${ }^{2}$ The goal of each of the validation experiments was to provide the most accurate description of each of the flowfields as possible. In order to successfully accomplish this goal, multiple instrument systems were utilized, allowing each to make its own contribution to documenting the velocity field as unambiguously as possible.

Each of the three cases involved some flowfield, or portion of the flowfield, being influenced by a synthetic jet actuator, also referred to as a synthetic jet or as a zero-mass-flux jet. The synthetic jet actuator was chosen as a representative actuator due to its widespread use in AFC work to date. Their popularity extends from the fact that they can be fabricated to a small size, they operate with zero net-mass, i.e, they synthesize a jet-like flowfield without being supplied by an external source of fluid, and they generate the type of disturbances required for AFC. The jet-like flow created by the synthetic jet, while zero-net-mass, imparts a finite vortical and momentum impulse to the fluid in order to synthesize a jet-like flow in the mean. A synthetic jet consists of a closed cavity, connected to the outer flowfield by an orifice. Typically, one of the walls of the closed cavity is an active element that is able to change the volume of the cavity. Thus in a synthetic jet, the flow through the orifice and out into the outer flowfield alternates between an exhaust and a suction cycle, driven by the contraction and expansion of a cavity internal to the actuator. During the exhaust cycle, a vortex ring is formed and moves away from the orifice under the action of self-induction. The cyclic nature of the cavity expansion and contraction causes a train of vortices to be formed, and this vortex train yields, in the mean, velocity profiles similar to that of a turbulent jet. ${ }^{3,4}$ In the open literature on AFC, one can find the synthetic jet being applied to achieve flow control on a circular cylinder, ${ }^{5-7}$ airfoils,,${ }^{8-11}$ and in internal flows. ${ }^{12,13}$

The three cases for the workshop are ordered in increasing levels of complexity with respect to the flow physics involved. The first of the three, Case 1 , was an isolated synthetic jet issuing into a quiescent environment. ${ }^{14}$ This case highlights the development of the vortex ring as the actuator goes through its exhaust cycle, the entrainment of the ambient fluid by the ring structure, and the movement of the ring away from the wall. The breakdown of the ring and the interaction between each ring and the previously formed ring are also key features that were documented. The second case, Case 2, builds upon the isolated synthetic jet of Case 1 by adding a crossflow, with no streamwise pressure gradient, for the synthetic jet to interact with. Here, the vortex ring formation is delayed and distorted by the crossflow. The path of the jet into the crossflow will start off initially being issued normally into the crossflow but will turn to be aligned with the crossflow as the two interact. The last case, Case 3 , involves the flow over a wall-mounted "hump" ${ }^{15}$ The flow here is characterized by a turbulent boundary layer flowing over a wall mounted disturbance, the hump, and adds the complexities of streamwise pressure gradients and a fixed separation location. Case 3 was examined in three conditions, the baseline flow, steady suction, and control of separation by synthetic jet actuation. It should be noted that while the cases are ordered in increasing flow physics complexity, Case 2 stands out as being the one case that cannot be approximated as being two-dimensional, it is inherently three-dimensional. Therefore, Case 2 may actually be the most challenging from a computational standpoint.

Formally, Case 2 examines the interaction of a single, isolated, synthetic jet and a fully turbulent zeropressure gradient boundary layer. The resulting flow has many of the characteristics that need to be modeled with fidelity if the results of the calculations are to serve as the basis for research and design with active flow control devices. These include the turbulence in the boundary layer, the time-evolution of the large vortical structure emanating from the jet orifice and its subsequent interaction with and distortion by the boundary layer turbulence, and the effect of the suction cycle on the boundary layer flow. The experimental setup is conceptually similar to that documented by Schaeffler. ${ }^{16}$ The interaction of a turbulent jet with a crossflow has been studied extensively experimental and computationally. The steady jet injected normal to a crossflow establishes the baseline for these types of flow. In the steady case, the jet is observed to transition, as it develops in the streamwise direction, from an orientation normal to the crossflow to being 
aligned with the crossflow. During this process, the jet column transforms into a pair of counter-rotating vortices. ${ }^{17}$ Additionally, there are other vortical structures near the jet exit that exist due to the interaction of the crossflow boundary layer and the orifice. ${ }^{18}$ The vorticity contained in the crossflow boundary layer is reorganized by its interaction with the jet, forming a number of structures in the wake of the jet, ranging from horseshoe vortex-like structures to vortices that connect the jet to the boundary layer along the wall. ${ }^{19}$ Adding an unsteady component to the jet, by some modulation of the jet velocity, increases the penetration of the jet into the crossflow ${ }^{20}$ and introduces some nomenclature for describing the degree of unsteadiness in the jet. Modulation on top of a mean flow creates the pulsed jet, ${ }^{20}$ turning the jet completely on and off yields a fully modulated pulsed jet, ${ }^{21}$ decreasing the frequency causes the formation of discrete vortex rings $^{22}$ with no mean jet flow, and finally increasing the frequency and adding a suction cycle, to achieve zero-net-mass flux, yields a synthetic jet. ${ }^{23-27}$

To document the flowfield of Case 2, with all of the richness described above, several measurement techniques were utilized. The upstream boundary conditions (in-flow conditions), and several phase-averaged velocity profiles were measured with a 3-component laser-Doppler velocimetry system. Phase-averaged velocity field measurements were made with both 2-D digital particle image velocimetry and stereo digital particle image velocimetry. Surface pressure measurements were made utilizing an electronically scanned pressure system. Dynamic pressure measurements were made in the actuator cavity. The actuator cavity temperature and the displacement of the actuator diaphragm were also documented. The focus of this paper will be more on the data acquisition and reduction of this data than is typical expected. However, a clear understanding of how the validation data was acquired and reduced is critical in order to compare CFD results with the experimental results. All of the data that is described in this paper is available from the Internet address given in the Appendix.

\section{Description of the Facility and Synthetic Jet Actuator}

This research effort was conducted in the NASA Langley 15-inch Low Speed Wind Tunnel of the Flow Physics and Control Branch. This tunnel is a closed-return atmospheric facility dedicated to basic flow physics research efforts. The tunnel has a maximum speed of $115 \mathrm{ft} / \mathrm{s}$ and a turbulence intensity of less than $0.13 \%$. The tunnel medium is air at sea level conditions. During this research effort, the tunnel was equipped with a suspended flat plate model that acts a splitter plate and facilitates the installation of the actuator within the plate model. The plate features an elliptical leading edge. Immediately downstream of the transition from the leading edge to the plate, there is a grit strip to trip the boundary layer of the plate and also grit strips at corresponding locations on the tunnel walls. The ceiling of this tunnel is adjustable at several locations down the length of the tunnel. The ceiling height was adjusted to create a zero pressure gradient over the plate starting from a station 36.5 inches $(927 \mathrm{~mm}$ ) downstream from the transition from the elliptical leading edge to the flat plate to a station 70.5 inches $(1791 \mathrm{~mm})$ downstream. The elliptical leading edge section of the splitter plate is 12.0 inches in length. The centerline of the jet was located 50.5 inches $(1283 \mathrm{~mm})$ downstream of the flat plate start. This results in the region of zero pressure gradient extending 56 jet diameters upstream of the jet centerline and 79 jet diameters downstream, as shown in Fig. 1 . These pressures were measured via a row of static pressure taps which are installed in the splitter plate, along the centerline of the tunnel, with a total of 15 taps upstream and 15 taps downstream of the jet centerline. A set of spanwise pressure taps, 16 in total, were located 8 jet diameters upstream of the jet centerline and another set of spanwise taps, also 16 in total, were located 8 jet diameters downstream. All of these taps were connected via short lengths of flexible Tygon tubing to two electronically-scanned pressure (ESP) pressure transducers, each with a full scale range of \pm 10 inches of water. The ESP modules were mounted inside the plate model and the measured pressures are referenced to the static pressure of the tunnel, as observed by a Pitot-static tube upstream of the splitter plate. The modules were automatically calibrated at regular intervals during a given test day. The error in the coefficient of pressure measurements are estimated to be $C_{p} \pm 0.002$. The pressure data was used to calculate the freestream velocity over the plate and to verify that 
the pressure gradient over the plate, in the area of interest, was zero. Flow visualization conducted in this tunnel suggests that the flow is two-dimensional in the zero pressure gradient region of the plate, with the exception of the region close to the side walls of the tunnel. ${ }^{13}$

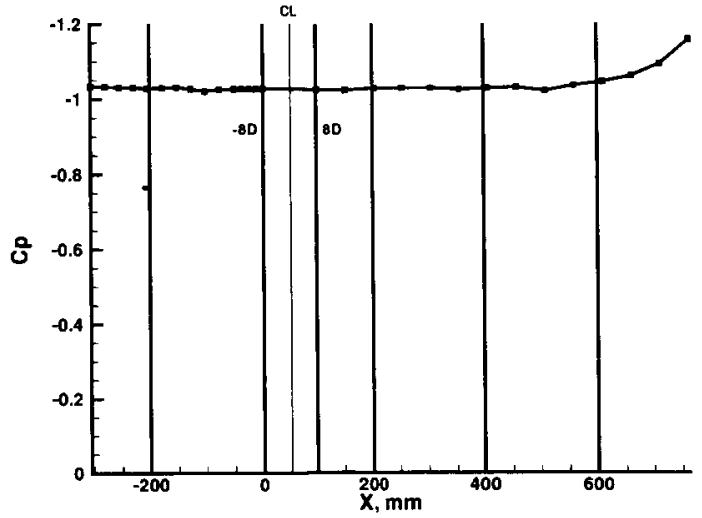

Figure 1. Pressure distribution down the centerline of the splitter plate, which corresponds to the centerline of the tunnel. $X$ is measured in the streamwise direction, and $X=0.00$ is the origin of the model coordinate system. The model coordinate system is shown schematically in Fig. 4.

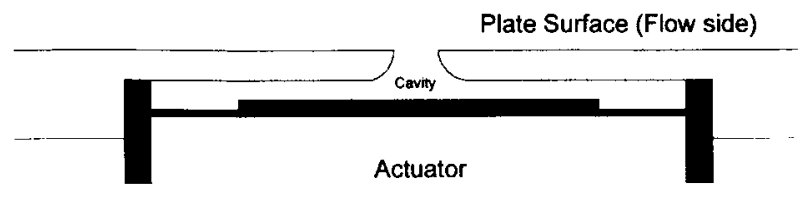

Figure 2. Schematic of the Synthetic Jet Actuator.

The synthetic jet utilized in this research effort featured a circular orifice, 0.25 inch $(6.35 \mathrm{~mm})$ in diameter. The throat of the orifice is smoothly tapered from a diameter of 0.60 inch $(15.2 \mathrm{~mm})$ on the inside cavity wall to the 0.25 inch diameter exit dimension. The upper cavity wall is 0.170 inch $(4.32 \mathrm{~mm})$ thick. The actuator design is shown schematically in Fig. 2. The active element of the actuator was the wall of the cavity opposite the exit of the jet, the bottom wall in Fig. 2. This wall was displaced electro-mechanically by a sinusoidal voltage. To document the in-situ performance of the actuator, the displacement of the actuator diaphragm was recorded, as was the cavity pressure and temperature. Two dynamic pressure transducers were installed in the interior of the cavity, an absolute pressure transducer and a differential pressure transducer. The differential pressure transducer had a range of $\pm 5.0 \mathrm{psi}$ and was referenced to the static pressure of the tunnel upstream of the splitter plate. A thermocouple was also located in the cavity to measure the cavity temperature.

\section{Measurement Systems}

It was a stated goal of the three validation experiments to measure their respective flowfields as unambiguously as possible. This required the use of redundant measurements of the velocity field. The velocity measurements to support Case 2 were carried out using a 3-component laser-Doppler velocimetry (LDV) system, a stereo digital particle image velocimetry (SDPIV) system, and a 2D digital particle image velocimetry (2D-PIV) system. The three measurement systems share some similar features and have some differences. None of the three systems actually measure fluid velocity. Each actually measures the velocity of tracer particles that are added to the flow. The LDV system can be regarded as a "point" measurement; the measurement is made within a $100 \mu \mathrm{m}$ sphere and is based on the velocity of a single particle. PIV systems are regarded as an area measurement, the measurement is made based on the displacement of multiple particles within an square area, typically $400-500 \mu \mathrm{m}$ on each side. The reader interested in further details is referred to one of the excellent review articles or monographs available on LDV $^{28,29}$ and PIV ${ }^{30-32}$ for more

\section{4 of 20}




\section{information.}

The LDV system utilized here was an orthogonal, crossed-fringe, fiber-optic probe configuration, with the probes mounted $90^{\circ}$ from one another. The 514.5, 496.5, and 476.5 nanometer wavelengths from an ArgonIon laser were used to measure the spanwise (V), vertical (W), and streamwise (U) velocity components, respectively within the tunnel. Bragg cells were utilized to provide directional measurement capability in all three velocity components. Both fiber optics probes used $750-\mathrm{mm}$ focal length lenses, which along with an input beam diameter of 3 to $4 \mathrm{~mm}$ for each of the six beams, generated a sample volume common to all three pairs that was calculated to be approximately $100 \mu \mathrm{m}$ in diameter and spherical in shape. The optical setup of the LDV system can be seen in Fig. 3. All velocity measurements were made in coincidence mode. Coincidence mode requires that a validated velocity signal be made independently in each of the three components within a very short time window, guaranteeing that the velocity signals come from a single particle traversing the measuring volume. The use of coincidence mode in the present research effort is required in order to obtain estimates for each of the six components of the Reynolds stress tensor.

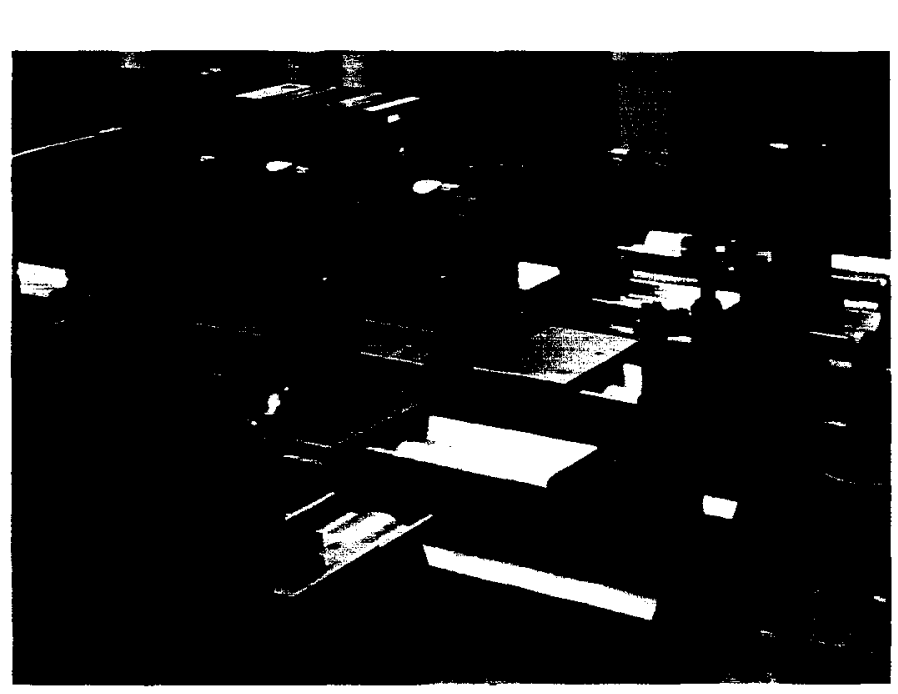

(a) Laser and Optical Configuration

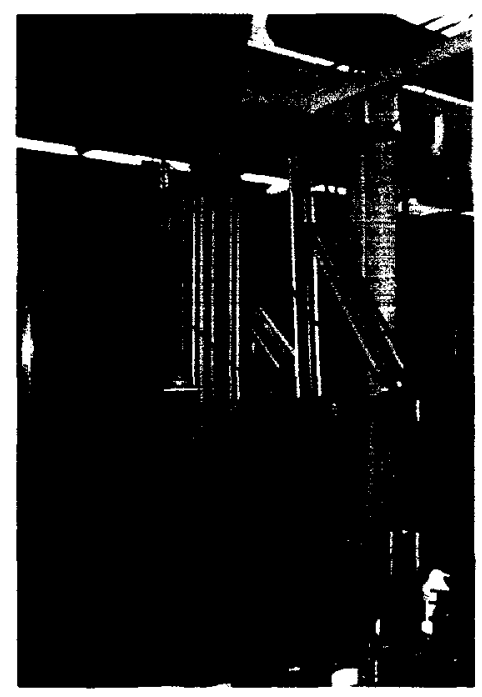

(b) Fiber Optic Bundles and Traversing System

Figure 3. The Laser-Doppler Velocimetry Setup.

Additionally, the actuator drive and sync signals were acquired with each trigger and stored if the velocity data was validated as being in coincidence. Consideration of the actuator drive and sync signals allows the LDV data, which is acquired at random intervals, to be reconstructed as a function of phase. The fiber optic probes were mounted on a three-axis traversing system, which utilized several motorized slides that provided 1 meter of travel in each of the three axes, with $1 \mu \mathrm{m}$ resolution in each direction. For the LDV portion of the experiment, the flow was seeded with mono-disperse, $0.86 \mu \mathrm{m}$ polystyrene latex (PSL) micro-spheres. The seed particles were suspended in 100-proof alcohol and atomized by a six-jet atomizer. The atomized particles were then introduced into the settling chamber of the tunnel. The particles were fabricated at NASA Langley using the technique described by Nichols. ${ }^{33}$

The two PIV configurations employed a standard commercial system which incorporated the timing control, image acquisition, data management and post-processing of both the 2D-PIV and the SDPIV data. The light source utilized was a pulsed, frequency-doubled $300 \mathrm{~mJ}$ Nd-YAG laser operating at $10 \mathrm{~Hz}$. For all the 
PIV data, the flow within the tunnel was seeded with mineral oil atomized to a typical particle size of $3.6 \mu \mathrm{m}$ by a six-jet atomizer. This particle sizing was experimentally verified by an aerodynamic particle sizer.

The seed was injected into the flow in the tunnel settling chamber. Standard sheet-forming optics were utilized and the cameras were positioned on opposite sides of the tunnel for the SDPIV data. Also for the SDPIV, the camera mounts allow for the required tilting of the lens, relative to the camera body, in order to satisfy the Scheimpflug condition. The light sheet orientation for the SDPIV data was in the spanwise direction and in the streamwise direction for the 2D-PIV data. The position of the sheet in the spanwise direction was controlled by a prism mounted on the same traversing system utilized by the LDV system. The light sheet thickness was typically $1.5 \mathrm{~mm}$ thick. The resolution of each camera was $1008 \times 1018$ pixels and the typical field of view was $40 \mathrm{~mm} \times 40 \mathrm{~mm}$ for the SDPIV and $18 \mathrm{~mm} \times 18 \mathrm{~mm}$ for the 2D-PIV. An interrogation window size of $32 \times 32$ pixels with $50 \%$ overlap was used for the PIV data. The PIV data was processed with a standard PIV interrogation technique by performing cross-correlations on equal-sized windows in both images.

The image acquisition and lasers for both PIV systems were synchronized with the drive signal of the actuator so that images could be acquired at a specific time delay after the beginning an actuator drive cycle. A specific delay for the laser pulsing and image acquisition corresponds to a specific phase of the actuator drive cycle. The same sync signal that the LDV system acquired was used as the trigger for the PIV signal. Within the PIV software, the timing delay was varied in order to acquire data at 36 specific phases of the synthetic jet actuator drive signal, equally spaced over both the exhaust and suction cycles. For the 2D-PIV data, the flowfield over the entire drive signal was recorded as 14,400 instantaneous velocity fields, 400 velocity field measurements at each of 36 specific delays, which results in the measurement of the velocity field every 10 degrees of phase. The 400 image pairs were acquired over a period of about 3 minutes. The images pairs for each phase were then processed using a standard PIV cross-correlation data reduction technique and then averaged to give the phase-averaged velocity field for that particular phase. 300 image pairs were utilized for each phase for the SDPIV data.

\section{Boundary Conditions}

The boundary conditions for Case 2 consist of the upstream, or in-flow, boundary condition, the geometry of the model and the tunnel itself, and the boundary conditions associated with the actuator, i.e., the diaphragm displacement, cavity pressure, and cavity temperature as a function of phase. The tunnel test condition was a Mach number in the test section over the plate of $\mathrm{M}=0.10$. The tunnel dimensions at the test section are 15.0 inches $(381 \mathrm{~mm})$ wide by $9.8 \mathrm{inch}(249 \mathrm{~mm})$ high (distance from the splitter plate to the top wall). The orifice diameter was $0.25 \pm 0.005$ inches $(6.35 \pm 0.13 \mathrm{~mm})$. The location of the jet orifice down the plate is specified in reference to the model coordinate system. The model coordinate system is shown schematically in Fig. 4 and its origin was established where the first line of spanwise pressure taps intersects the line of centerline pressure taps. This location is 60.5 inches $(1537 \mathrm{~mm})$ from the leading edge of the plate. In the model coordinate system, the centerline of the jet orifice is located at $2.00 \pm 0.020$ inches $(50.8 \pm 0.50 \mathrm{~mm})$. Moving from the origin of the model coordinate system to the jet centerline in the streamwise direction defines the $\mathrm{X}$ coordinate axis, the $\mathrm{Y}$ coordinate axis is in the spanwise direction and the $\mathrm{Z}$ coordinate axis is in the vertical direction. The corresponding instantaneous velocity components will be $\mathrm{u}, \mathrm{v}$, and $\mathrm{w}$. The mean velocity components will be denoted by $U, V$, and $W$ and the six turbulent stresses by $\overline{u^{\prime} u^{\prime}}, \overline{v^{\prime} v^{\prime}}, \overline{w^{\prime} w^{\prime}}, \overline{u^{\prime} v^{\prime}}, \overline{u^{\prime} w^{\prime}}, \overline{v^{\prime} w^{\prime}}$. From this point forward, distances in the streamwise, $\mathrm{X}$, direction will be stated in millimeters or, at times, jet diameters from the jet centerline. The key locations are: $\mathrm{X}=44.45 \mathrm{~mm}(-1 \mathrm{D}), \mathrm{X}=50.80 \mathrm{~mm}$ ( OD, CL), $\mathrm{X}=57.15 \mathrm{~mm}$ (1D), $\mathrm{X}=63.50 \mathrm{~mm}$ ( 2D), $\mathrm{X}=69.80 \mathrm{~mm}$ (3D), and $X=76.20 \mathrm{~mm}$ ( $4 \mathrm{D})$. All velocity components and turbulence quantities presented hereafter, unless noted otherwise, have been non-dimensionalized by the freestream velocity, or the freestream velocity squared. 
While the atmospheric conditions varied on a daily basis, the conditions were never far from standard atmospheric conditions at sea level in a wind tunnel vented to the atmosphere, in a temperature-controlled room. These conditions are given in Table 1 .

\begin{tabular}{|l|l||l|}
\hline Freestream Mach Number & $M_{\infty}$ & 0.10 \\
Atmospheric Pressure & $P_{a t m}$ & $101,325 \mathrm{~Pa}$ \\
Atmospheric Temperature & $T_{\infty}$ & $23.9^{\circ} \mathrm{C}\left(75^{\circ} \mathrm{F}\right)$ \\
Density & $\rho_{\infty}$ & $1.185 \mathrm{~kg} / \mathrm{m}^{3}$ \\
Viscosity & $\mu_{\infty}$ & $18.4 \times 10^{-6} \mathrm{~kg} / \mathrm{ms}$ \\
\hline
\end{tabular}

Table 1. Atmospheric Conditions Observed for Case 2

The upstream boundary conditions were measured at station $\mathrm{X}=0.0 \mathrm{~mm}$ in the model coordinate system. A complete description of the state of the turbulent boundary layer was measured using the 3-component laserDoppler velocimetry system to be described in the following section. All six components of the turbulent stress tensor were documented and presented as the upstream boundary condition. The velocity means and the turbulent stress components were compared to similar values available in the open literature ${ }^{34}$ and found to be in good agreement, as shown for the mean profile of the freestream component of the velocity, $\mathrm{U}$, and some of the turbulent stresses, $\overline{u^{\prime} u^{\prime}}, \overline{v^{\prime} v^{\prime}}, \overline{w^{\prime} w^{\prime}}, \overline{u^{\prime} w^{\prime}}$ in Fig. 5 .

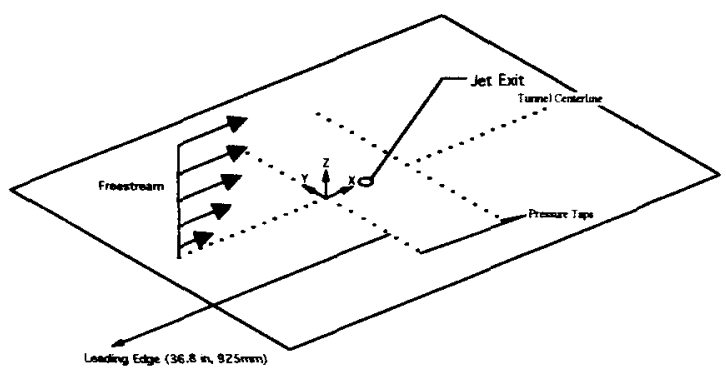

Figure 4. Schematic of the Model Coordinate System.

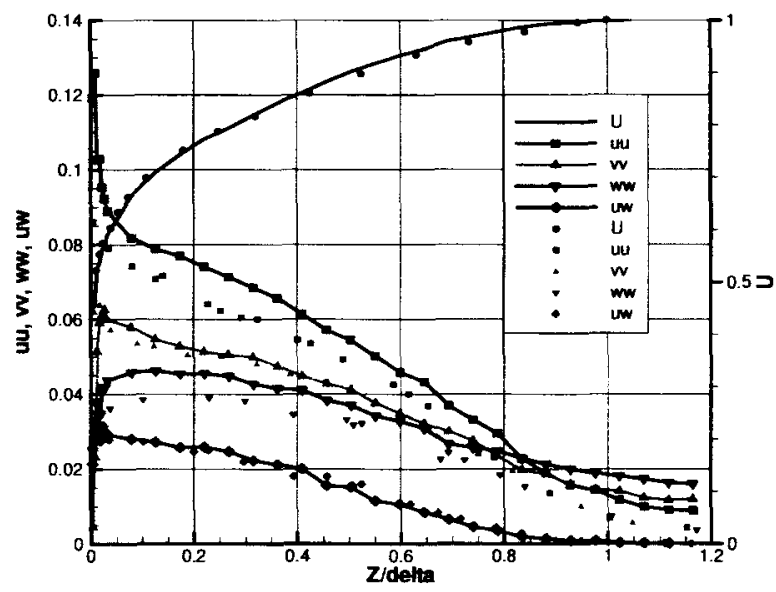

Figure 5. Upstream Boundary Condition (UBC) compared to the data of Klebanoff. ${ }^{34}$ Solid symbols without lines are the Klebanoff data. Data with empty symbols and lines are the UBC. All velocity data is non-dimensionalized by the freestream velocity.

The final component of the boundary conditions are the boundary conditions associated with the actuator itself, i.e., the diaphragm displacement and the cavity pressure and temperature as a function of phase. The phase-averaged diaphragm displacement and cavity pressure can be seen in Fig. 6, along with the actuator drive signal. The presentation of all of the subsequent velocity measurements utilize this same phase reference. The diaphragm displacement is relative to the nominal cavity depth when the tunnel is on condition. This value is $1.68 \mathrm{~mm}$. The cavity temperature was observed not to be a function of phase. The cavity temperature was elevated from the tunnel temperature by $2^{\circ} \mathrm{F}\left(77^{\circ} \mathrm{F}\right)$. 


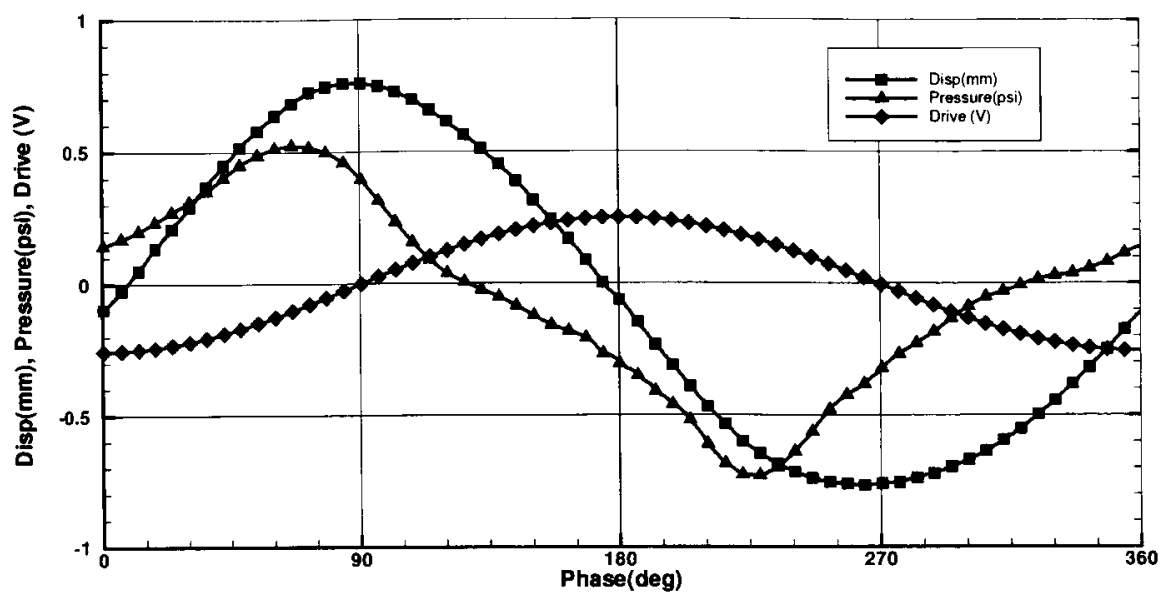

Figure 6. Diaphragm displacement, drive signal and cavity pressure as a function of phase for the Synthetic Jet Actuator.

\section{Data Reduction, Uncertainty, and Comparison}

As discussed previously, the LDV system and the PIV system utilize different techniques to measure velocity. The LDV is a point measurement based on the velocity of a single particle. The PIV system is an area measurement, based on the displacement of multiple particles in a small area illuminated by a laser sheet. The two measurement systems also see the phase of the jet in different ways. The PIV system can be triggered, to a high degree of accuracy, to acquire its images at a particular time. This means that the PIV system can be triggered when the actuator drive signal is exactly at $30^{\circ}$ phase. The LDV system, in contrast, acquires data at random intervals, corresponding to whether there is a particle in the sample volume that generates a valid signal in all three components. We need to sort the LDV data by phase, after it is acquired, in order to phase average the data. This must be done by binning some range of phase angles together, in order to get enough points to generate meaningful statistics. So the LDV system provides information that is at a point in space, but "smeared" in time, while the PIV system is at a "point" in time, by "smeared" in space.

\section{A. LDV Data Reduction}

It is the nature of the LDV data that the velocity is randomly sampled in time. Valid velocity measurements are made only when there is a single particle in the sample volume which scatters sufficient light to generate a valid signal in each of the three velocity components. So once the data is acquired, it must be sorted based on the phase of the actuator at the time of acquisition. The LDV data system acquires, along with the coincident velocity data, data for the drive signal of the actuator and a sync signal, a square wave in phase with the drive signal. Analysis of the value of the drive signal and the value of the sync signal allows for the phase angle at the time of acquisition to be calculated. Once a phase angle is assigned to all the LDV data, the data can be sorted into bins according to phase angle. Each bin represents data with a phase angle in a certain range, the width of the bins utilized here was $10^{\circ}$. For example, all the data from $0^{\circ}$, inclusive, to $10^{\circ}$, exclusive, was grouped in a single bin. The data was then clipped based on the number of samples in the bin and Chauvenet's criterion..$^{35}$ The data could then be averaged and the turbulence quantities calculated. This then becomes the phase-averaged data for this bin and was assigned a phase angle in the center of the bin, in this example $5^{\circ}$. Analysis of the average phase angle in all the bins provides a value very close to 
the halfway point indicating that there is not a bias towards one side of the bin or the other. Once the data in each individual bin has been phase-averaged, the data from the 36 bins, or each individual phase, was averaged together to calculate the long-time average profile, the long-time mean. The long-time average velocity profiles derived from the LDV data are shown in Fig. 7.

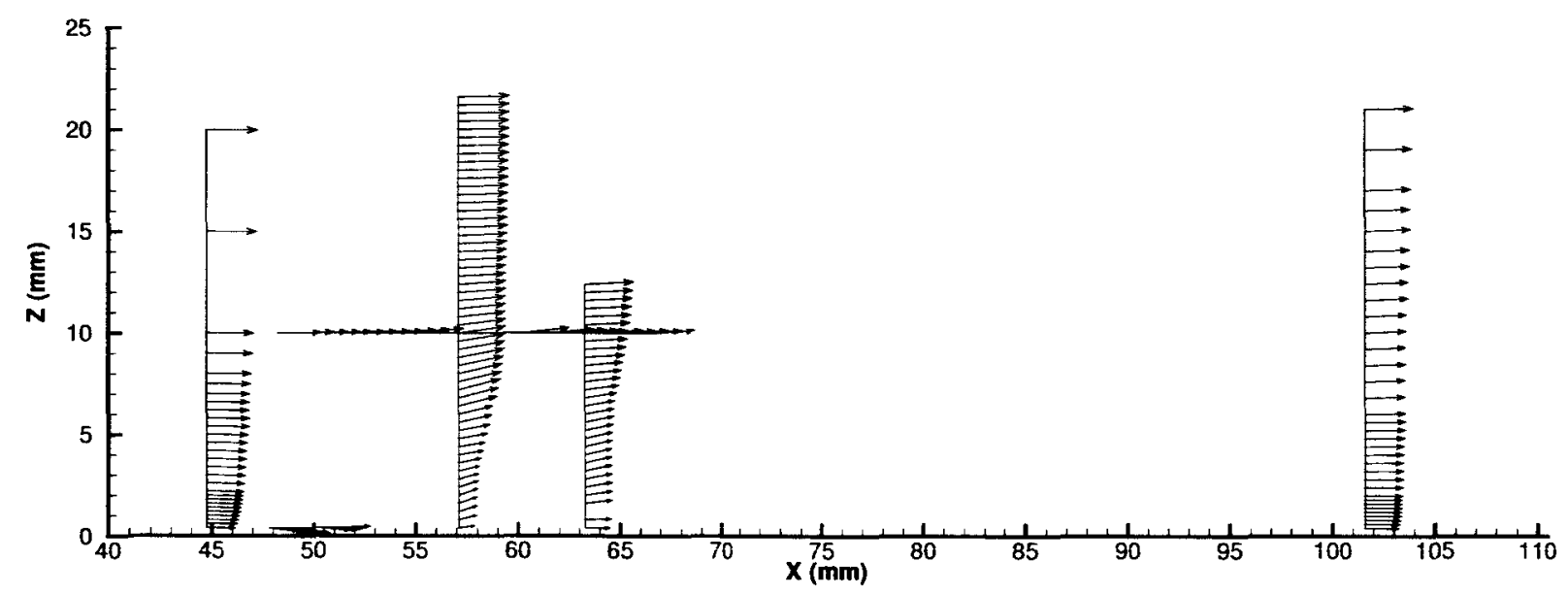

Figure 7. Long-Time Average Velocity Profiles from the LDV data. The jet exit is located from $47 \leq X \leq 54$ and the upstream boundary condition of Fig. 5 would be located at $X=0$.

The phase-averaged statistics, the mean velocity and turbulence quantities, for each bin of the LDV data were computed using weighted averages. ${ }^{28}$ Specifically, the following formulations were used:

$$
\begin{aligned}
& U=\frac{\sum_{N} u_{i} g_{i}}{\sum_{N} g_{i}} \quad \overline{u^{\prime} u^{\prime}}=\frac{\sum_{N}\left(u_{i}-U\right)^{2} g_{i}}{\sum_{N} g_{i}} \quad \overline{u^{\prime} v^{\prime}}=\frac{\sum_{N}\left(u_{i}-U\right)\left(v_{i}-V\right) g_{i}}{\sum_{N} g_{i}} \\
& V=\frac{\sum_{N} v_{i} g_{i}}{\sum_{N} g_{i}} \quad \overline{v^{\prime} v^{\prime}}=\frac{\sum_{N}\left(v_{i}-V\right)^{2} g_{i}}{\sum_{N} g_{i}} \quad \overline{v^{\prime} w^{\prime}}=\frac{\sum_{N}\left(v_{i}-V\right)\left(w_{i}-W\right) g_{i}}{\sum_{N} g_{i}} \\
& W=\frac{\sum_{N} w_{i} g_{i}}{\sum_{N} g_{i}} \quad \overline{w^{\prime} w^{\prime}}=\frac{\sum_{N}\left(w_{i}-W\right)^{2} g_{i}}{\sum_{N} g_{i}} \quad \overline{u^{\prime} w^{\prime}}=\frac{\sum_{N}\left(u_{i}-U\right)\left(w_{i}-W\right) g_{i}}{\sum_{N} g_{i}}
\end{aligned}
$$

When free-running weights are used, i.e. $g_{i}=1$, the calculated statistical quantities revert to their standard definitions, e.g. U is the arithmetic mean of the $u_{i}$ 's. Use of this type of weighting scheme results in values that are an upper bound on the precision error. ${ }^{28}$

Since all three components of velocity were acquired and the use of coincidence mode yields a nearly spherical sample volume, the use of a weighting function based on the velocity magnitude is attractive and appropriate for use. In this case, the weighting function, $g_{i}$, will be the inverse of the magnitude of the velocity vector,

$$
g_{i}=\frac{1}{\sqrt{u_{i}^{2}+v_{i}^{2}+w_{i}^{2}}}
$$


Both the free-running and the velocity magnitude weighting functions were considered here. The mean velocity values calculated by using the free-running weights were higher than the means calculated by the velocity magnitude weights indicated that there might be some velocity bias in the data. Therefore to minimize this effect, the velocity magnitude weights were adopted for use and were applied to all of the LDV data.

Once all of the LDV data has been phase-averaged, the effect of the jet on the flow can begin to be investigated. In Fig. 8, the velocity profile one diameter upstream of the jet centerline (-1D) is presented as a function of phase and with the vectors colored by the value of $\overline{u^{\prime} w^{\prime}}$. At this station, the effect of the jet is felt most strongly during the suction portion of the cycle. During that time, the velocity profile near the wall becomes fuller indicating that the near wall fluid is accelerated by the suction cycle of the jet. The effect of the exhaust cycle is less than the suction cycle at this station. An additional vertical component of velocity is observed throughout the profile as the fluid prepares to flow over the top of the jet, which appears as an obstruction to the oncoming flow.

At the station one diameter downstream of the jet centerline $(+1 \mathrm{D})$, the situation is quite different. Here the fluid expelled from the jet during the exhaust cycle passes through this station with a marked change in the profiles. In Fig. 9, the velocity profile one diameter downstream of the jet centerline is presented as a function of phase and again with the vectors colored by the value of $\overline{u^{\prime} w^{\prime}}$. The exhaust phase is marked by a large vertical component of velocity that passes through this station with corresponding large values of $\overline{u^{\prime} w^{\prime}}$. The suction cycle also sees a large vertical component of velocity as the fluid displaced from the wall by the jet moves back to the wall as the exhaust cycle of the jet collapses.

\section{B. PIV Data Reduction}

As previously described in Section III, the PIV system can be configured to acquire images at an exact phase of the actuator drive signal, so acquiring the data for each phase is not as problematic as it is for the LDV system. All of the image pairs are acquired and then processed using a standard PIV cross-correlation technique. The instantaneous velocity filed are validated and any missing vectors in the field are replaced by interpolation. The instantaneous velocity fields are then averaged to produce the phase-averaged velocity field and statistics for that phase. No additional processing on the instantaneous fields was done. The PIV measurements were also taken at $10^{\circ}$ intervals, so once all 36 phase averages were calculated, they were averaged together to generate the long-time mean velocity field.

The 2D-PIV data can provide some interesting insight into the interaction of the synthetic jet and the crossflow. In Fig. 10, the velocity field at a phase of $0^{\circ}$ is presented. This phase is during the suction cycle and we can see the strong influence that the jet has on the flow upstream of the actuator. All of the flow that is filling the expanding cavity has a tendency to come from upstream of the jet as shown by the instantaneous, in a phase-averaged sense, streamline pattern. In fact, the pattern shows the dividing streamline and half-saddle that forms on the wall immediately downstream of the aft edge of the jet, which mark the segregation of the fluid that will tend to flow towards the actuator from the fluid that will tend to flow over the actuator.

In Fig. 11, the velocity field at a phase of $130^{\circ}$ is presented. At this phase, the jet is near the peak of the exhaust cycle. The jet column can already be seen to be turning in the direction of the freestream by the edge of the frame. This height is only about half the undisturbed boundary layer thickness, illustrating that the interaction of the synthetic jet and the crossflow remains contained in the boundary layer. This was one of the requirements for the experiment.

The long-time mean of the velocity field was calculated by averaging, through one complete cycle, the individual phase-averaged velocity fields, as shown in Fig. 12 for data derived from the 2-D PIV system. We can see that in the long-term mean, the synthetic jet does appear as a steady jet which turns in the direction of the freestream close to the wall. Another long-time mean feature of the flowfield is the appearance of 


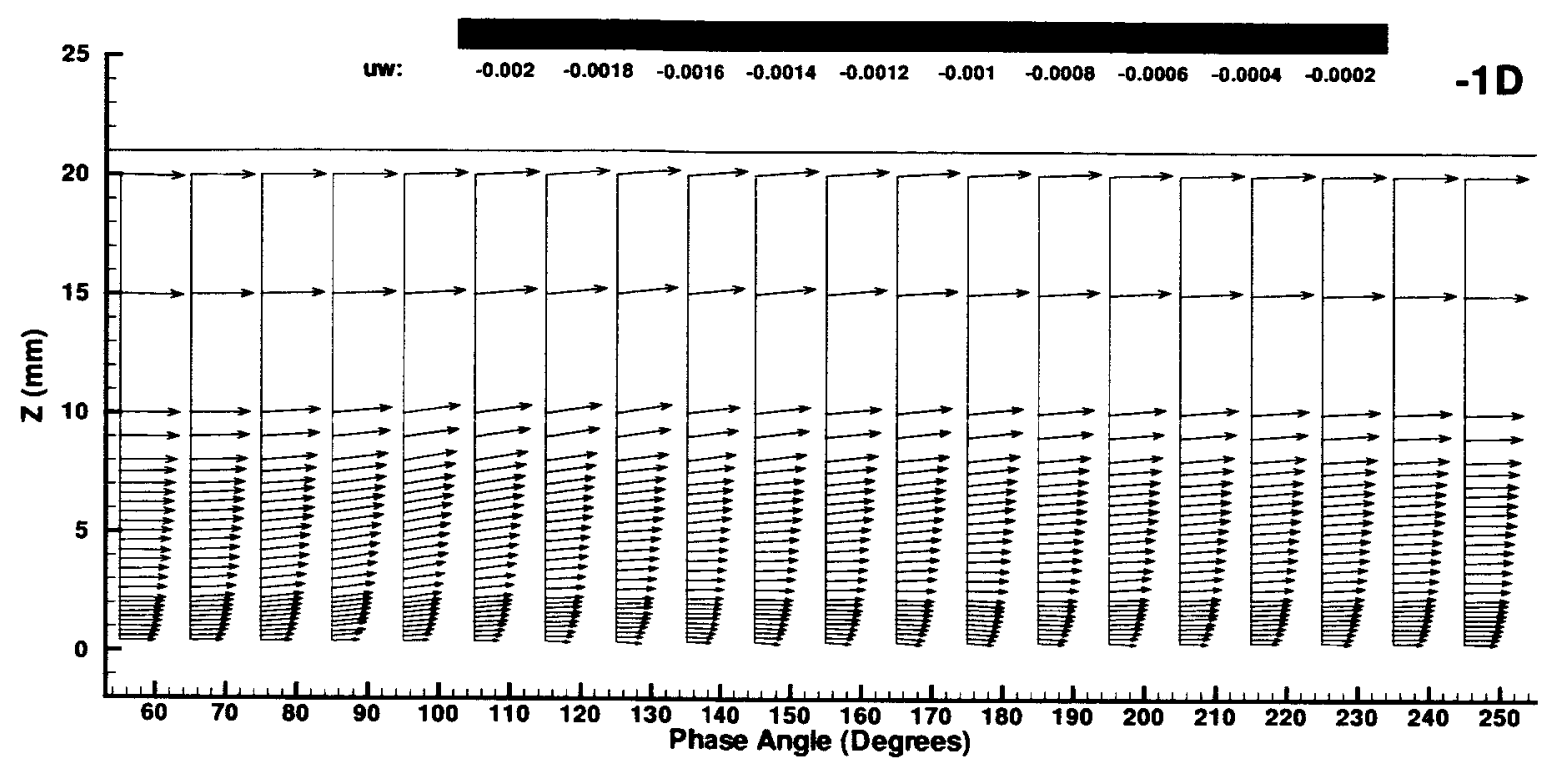

(a) Exhaust portion of the cycle

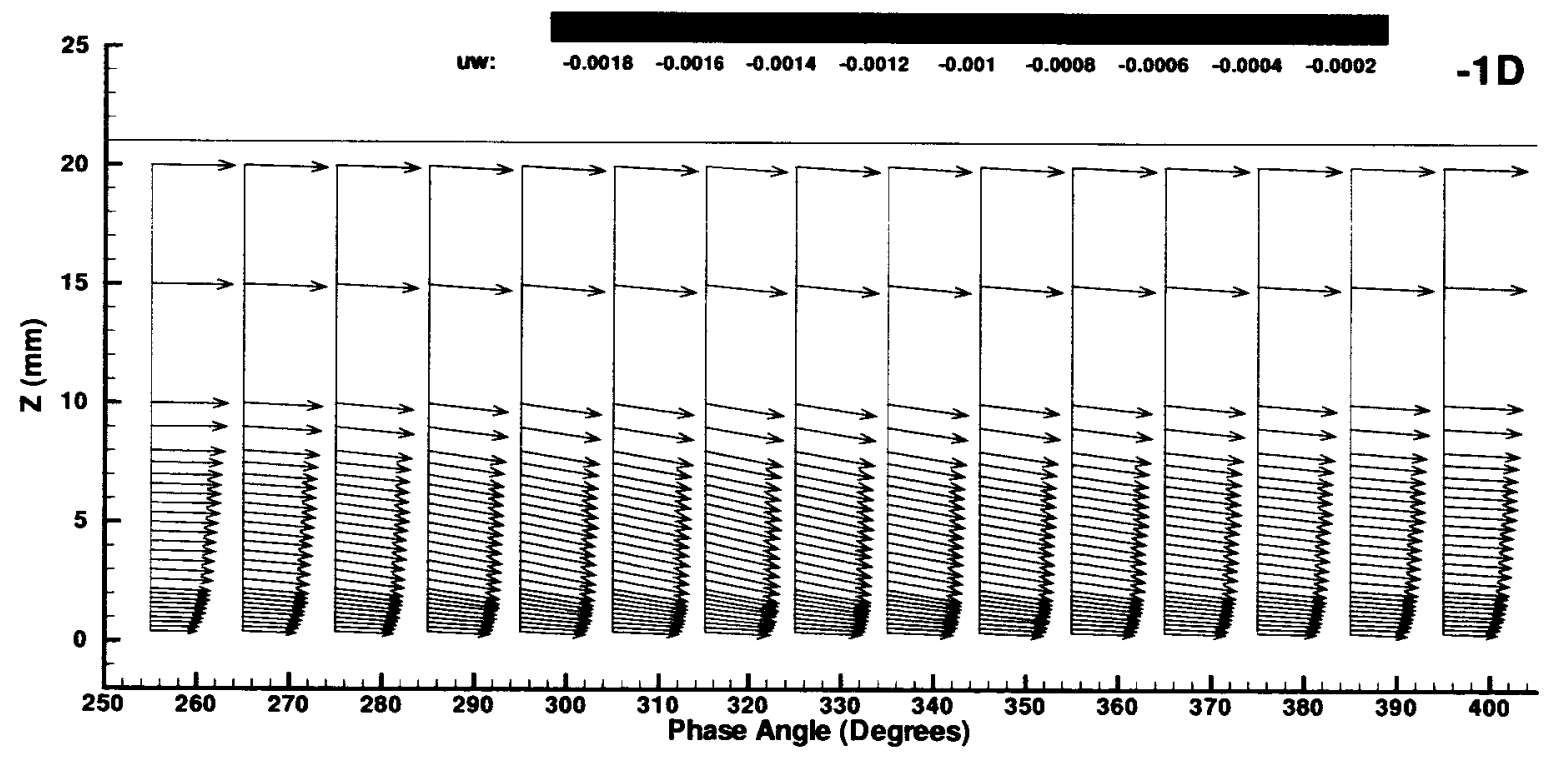

(b) Suction portion of the cycle

Figure 8. Phase-Averaged LDV Velocity Profiles at $x=44.45 \mathrm{~mm}(-1 D)$ as a function of phase angle. Thin horizontal line at $\approx 21 \mathrm{~mm}$ is the height of the undisturbed boundary layer at this station. 


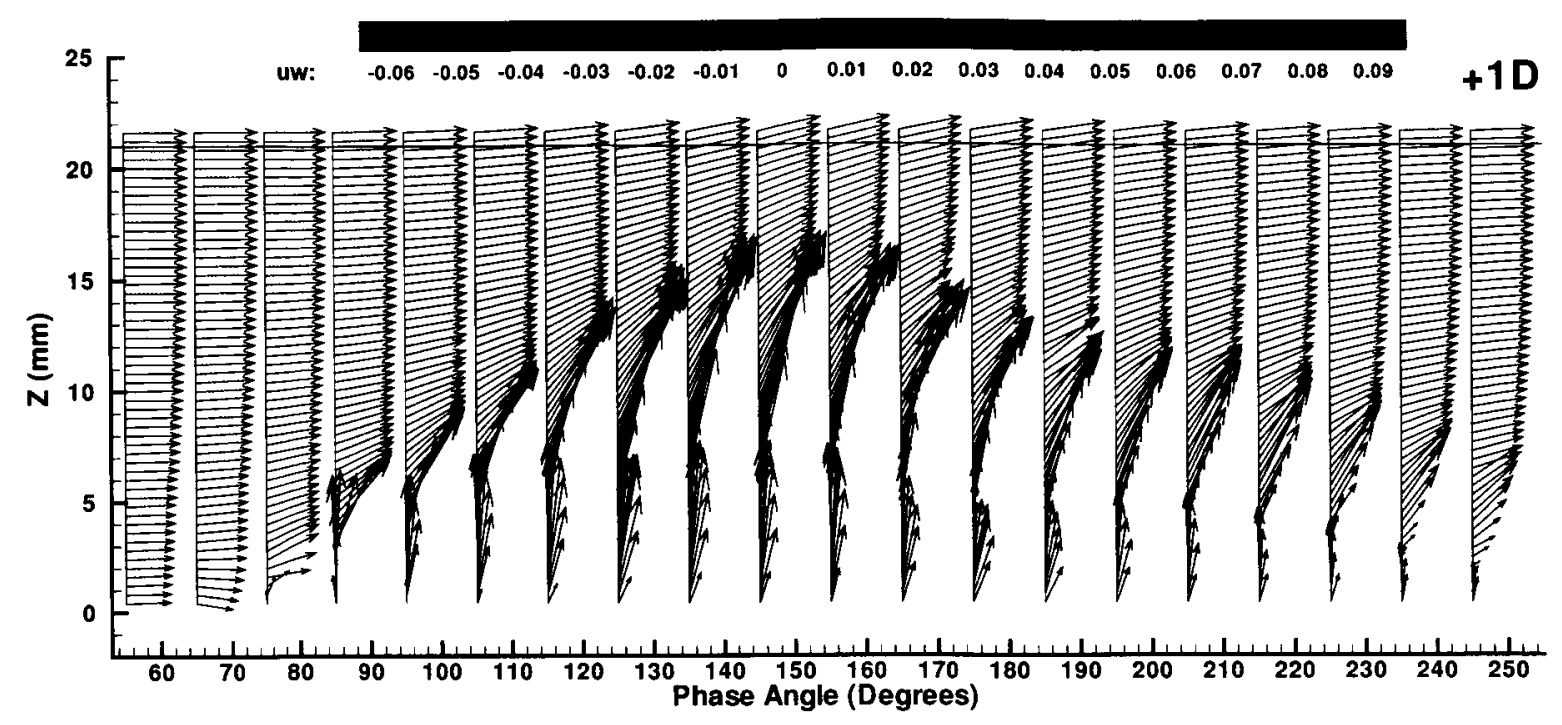

(a) Exhaust portion of the cycle

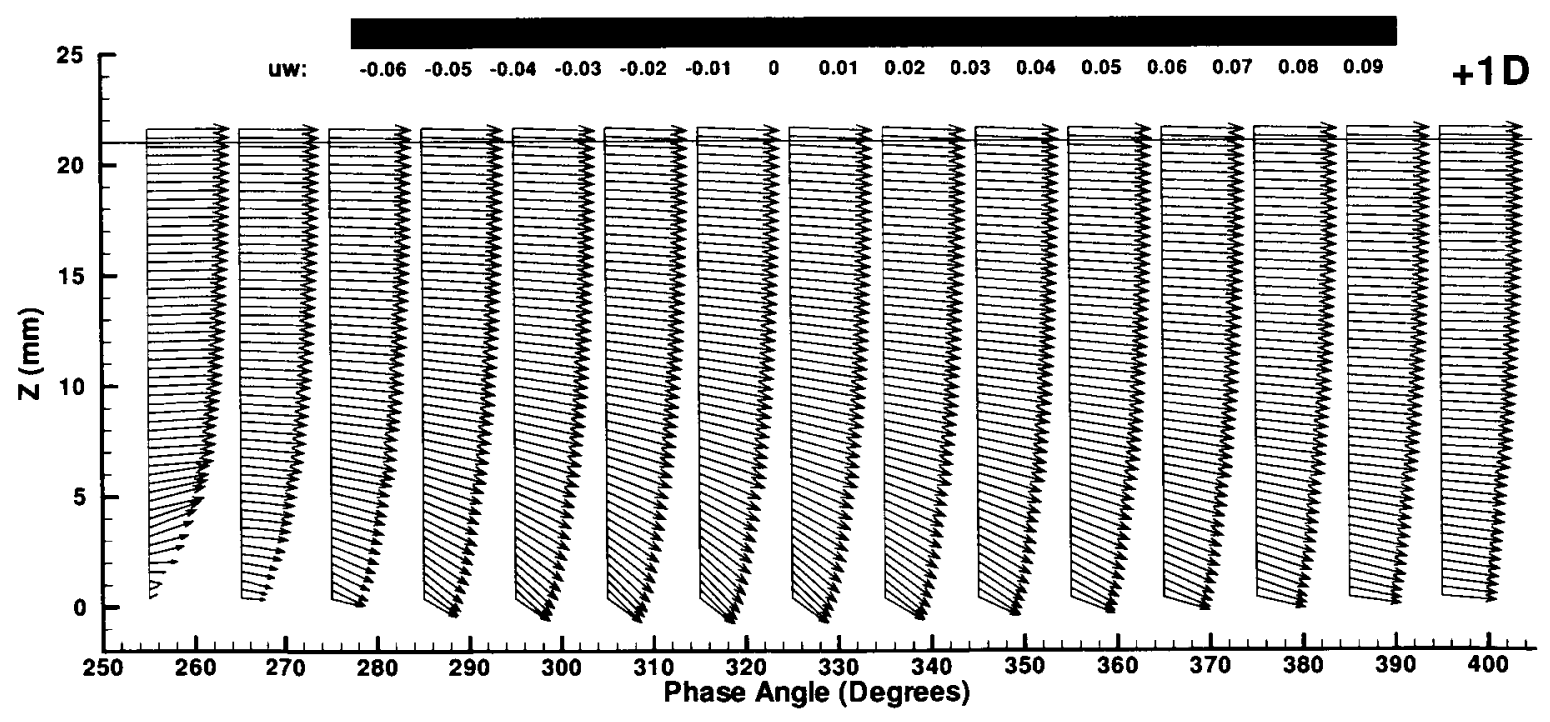

(b) Suction portion of the cycle

Figure 9. Phase-Averaged LDV Velocity Profiles at $x=57.15 \mathrm{~mm}(+1 D)$ as a function of phase angle. Thin horizontal line at $\mathrm{Z}=21 \mathrm{~mm}$ is the height of the undisturbed boundary layer at this station. 


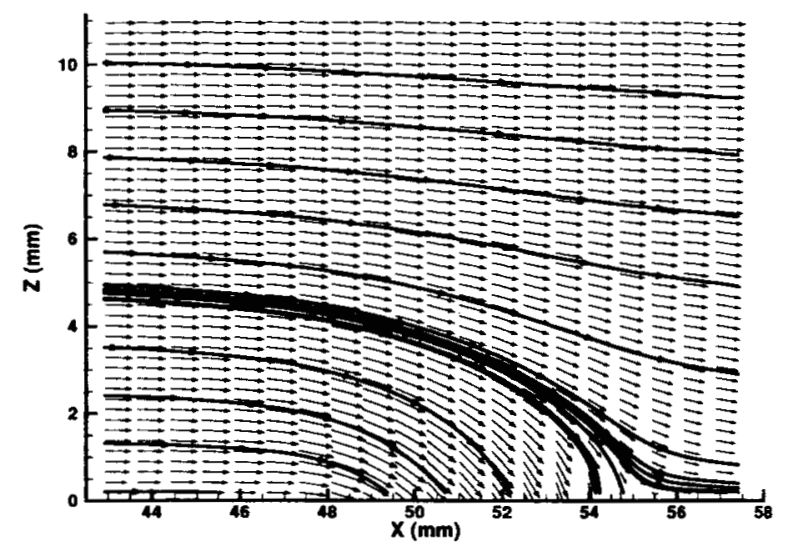

Figure 10. PIV Velocity field during the suction part of the cycle. Instantaneous streamlines indicate that a half-saddle is formed near the aft end of the jet exit during the suction cycle $\left(0^{\circ}\right.$ Phase).

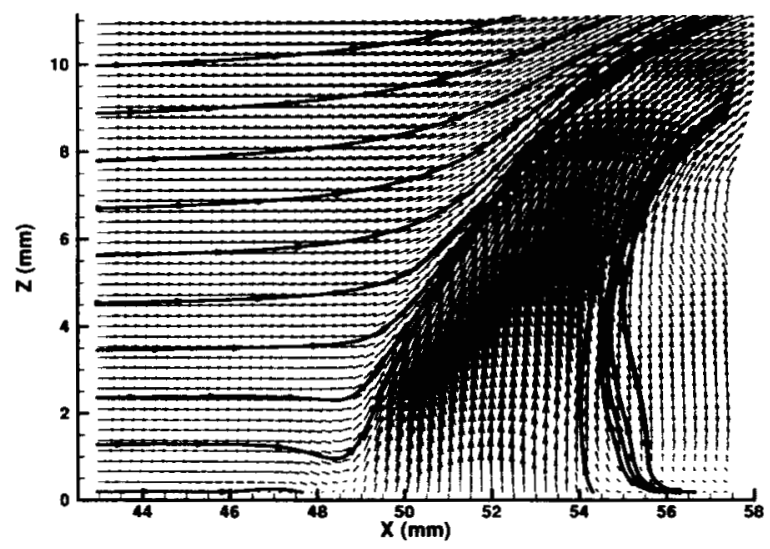

Figure 11. PIV Velocity field during the exhaust part of the cycle $\left(130^{\circ}\right.$ Phase).

counter-rotating vortices in a spanwise plane. This spanwise plane, measured with the SDPIV system, is $4 \mathrm{D}$ downstream of the jet centerline. The development of a counter-rotating vortex pair is very similar to what occurs in the interaction of a steady jet and a crossflow.

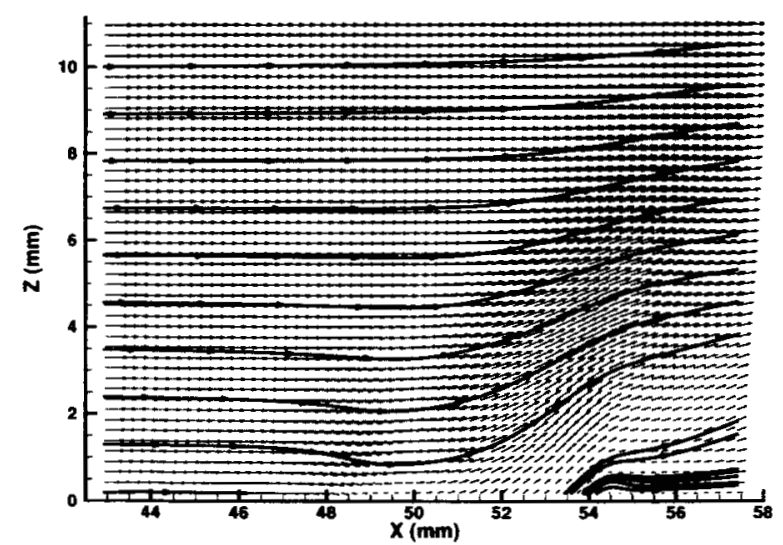

Figure 12. Long-time mean velocity field (2D-PIV measurements).

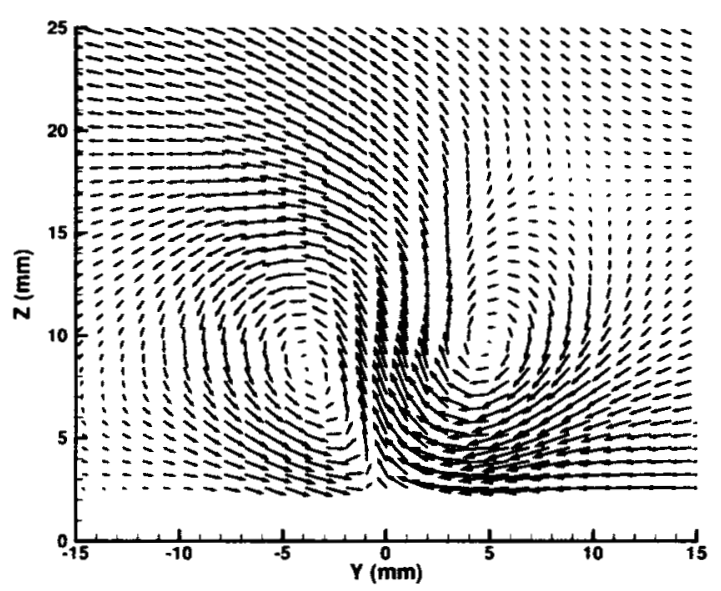

Figure 13. Counter-rotating vortices in the long-time mean flow $4 D$ downstream of the jet centerline (SDPIV measurements).

\section{Uncertainty}

In truth, the mean velocities and turbulent stresses presented herein are, at best, estimates of the actual statistical values. The assignment of experimental uncertainty to each of the mean velocities and turbulent stresses is a way of providing a range on each of the measurements that has a high degree of confidence of containing the true value. This range is usually specified by a degree of confidence, or a set of odds, that the true value does indeed lie in the given range. A confidence of $95 \%$, or $20: 1$ odds, will be used here. 
The total uncertainty is calculated by a quadrature of the bias error and the precision error. ${ }^{35}$ The bias error is composed of the errors that are present in every single measurement that an instrument makes and are not effected by the number of samples taken. They are the errors that can be minimized, but not eliminated, by careful setup, alignment, and choice of instruments. For the LDV system, one of the biggest contributors to the bias error is the accuracy with which the half-beam angle is know for all three beam pairs. (In a LDV system, each velocity component is calculated by $v=f_{d} \lambda /(2 \sin \kappa)$, where $f_{d}$ is the Doppler frequency and $\lambda$ is the wavelength of the laser light, both of which are known with low uncertainty. Therefore, the half-beam angle, $\kappa$, must also be known with a low uncertainty. ${ }^{28}$ The half-beam angle was measured through an in-situ calibration, thus minimizing the uncertainty of the half-beam angle for each of the beam pairs. The bias error for the LDV data is estimated to be $\pm 0.5 \%$ of the instantaneous velocity measurement, $\pm 0.5 \% u_{i}$. For the 2D-PIV system, the bias error is estimated to be $\pm 1.3 \%$ of the instantaneous velocity measurement, $\pm 1.3 \% u_{i}$.

The fact that the data taken here represents a limited number of samples of a random process requires that the effect of the sample size, $\mathrm{N}$, on the uncertainty in our estimate of the velocity means and turbulence quantities be considered. This is the precision error portion of the uncertainty and is related to the variances of each of the estimated velocity means and turbulent stresses. Once the estimator variances are known, the precision error can be evaluated, at 20 to 1 odds, as being 2.0 times the square root of the variance. The precision error can then be combined with the bias error through quadrature.

Following the work of Benedict and Gould ${ }^{36}$ the estimator variances for each of the mean velocities and turbulence quantities were calculated as follows:

$$
\begin{aligned}
& \sigma_{U}^{2}=\frac{\overline{u^{\prime} u^{\prime}}}{N} \quad \sigma_{\overline{u^{\prime} u^{\prime}}}^{2}=\frac{\overline{u^{\prime}}-{\overline{u^{\prime} u^{\prime}}}^{2}}{N} \\
& \sigma_{\frac{2}{u^{\prime} v^{\prime}}}=\frac{\overline{u^{\prime 2} v^{\prime 2}}-{\overline{u^{\prime} v^{\prime}}}^{2}}{N} \\
& \sigma_{V}^{2}=\frac{\overline{v^{\prime} v^{\prime}}}{N} \\
& \sigma_{\overline{v^{\prime} v^{\prime}}}^{2}=\frac{\overline{v^{\prime 4}}-{\overline{v^{\prime} v^{\prime}}}^{2}}{N} \\
& \sigma \frac{2}{u^{\prime} w^{\prime}}=\frac{\overline{v^{\prime 2} w^{\prime 2}}-{\overline{v^{\prime} w^{\prime}}}^{2}}{N} \\
& \sigma_{W}^{2}=\frac{\overline{w^{\prime} w^{\prime}}}{N} \\
& \sigma \frac{2}{w^{\prime} w^{\prime}}=\frac{\overline{w^{\prime}}-{\overline{w^{\prime} w^{\prime}}}^{2}}{N} \\
& \sigma_{\overline{u^{\prime} w^{\prime}}}^{2}=\frac{\overline{u^{\prime 2} w^{\prime 2}}-\overline{u^{\prime} w^{\prime}}}{N}
\end{aligned}
$$

These relations illustrate that the higher the turbulence level, the higher the uncertainty in the evaluation of the corresponding mean velocity, for a given number of samples. This effect is alluded by Uzol and $\mathrm{Camci}^{37}$ in their analysis of the influence of turbulent intensity on the accuracy of ensemble averaged PIV measurements. During the jet exhaust, the turbulence levels, the variances seen in the observation of the instantaneous velocities, become very high. This can be seen by looking at the raw LDV data after it has been sorted by phase. If we consider the velocity in the streamwise direction, $u$, the spread of that data at a given phase is related to the variance, which in this case is also the turbulent stress, $\overline{u^{\prime} u^{\prime}}$. This spread can be seen in Fig. 14. For the phases not during the exhaust cycle of the jet, the spread of the data is modest and indicative of the underlying turbulence levels in the boundary layer. For phases during the exhaust cycle, the spread is much larger. The underlying turbulence and cycle-to-cycle variations in the jet formation process contribute to a increased spread of the data and calculated mean velocities for these phases will have a higher uncertainty associated with them. 


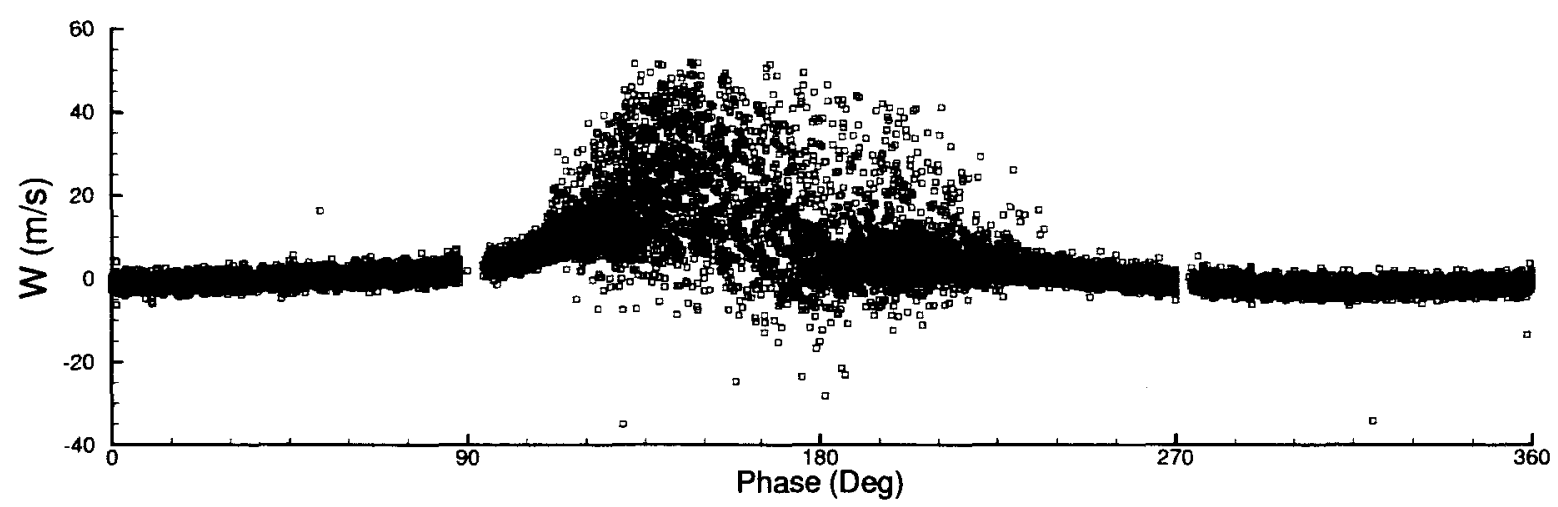

Figure 14. LDV data for $(57.1,0.0,10.4)$ after the phase data has been reconstructed but before any of the points are clipped. The gaps near $90^{\circ}$ and $270^{\circ}$ are artifacts of the phase reconstruction.

\section{Comparison of the LDV and the PIV data}

In order to compare the velocity measurements made by the LDV system with those made by the PIV system, data from common locations in both datasets were examined as a function of phase. At a location 1D downstream (X $=57.1 \mathrm{~mm})$, and $14.0 \mathrm{~mm}$ off the wall, we are above the disturbance of the jet. A comparison of the LDV data and the PIV data at that location, as shown in Fig. 15, shows an acceptable agreement between the two. The 2D-PIV data tends to underestimate the velocity with respect to the LDV, which may be due to the larger tracer particles used in the PIV portion of the experiment.

If we move closer to the wall at the 1D station, to $10.4 \mathrm{~mm}$ off the wall, the agreement worsens. This location, as shown in Fig. 16, is in the middle of the interaction of the jet and the crossflow. During the exhaust cycle, the LDV and the PIV data show different $U$ velocity signatures. The LDV U signature indicates that the streamwise velocity component, $U$, increases during the interaction with the jet and then decreases slightly from the non-interaction, or suction cycle, value $(U \approx 0.90)$. The PIV U signature shows an opposite effect, the $\mathrm{U}$ component decreases through the interaction and then slightly increases before moving back to its value during the suction cycle. The $\mathrm{W}$ signature from the PIV and LDV have a closer correspondence to one another, but there are some discrepancies. The LDV W signature is slightly wider at its peak than the PIV W signature and there is a second feature in the LDV signature that is not present in the PIV signature $(180<$ Phase $<230)$.

In order to determine if the discrepancy was some how an artifact of the processing or the statistical properties of the data, several alternative processing techniques and analyses were performed. To see if the disagreement was related to the bin size of the LDV processing, the effect of reducing the bin size was investigated. As shown in Fig. 17, the amount of noise on the $U$ velocity signature is increased because of the reduction in the number of samples in each bin as the total number of bins is increased, but increasing the number of bins does not change the basic shape of the signature. To see if there was a sufficient number of samples to achieve a statistically stationary value for the means, the effect of the sample, or ensemble, size was investigated. As shown in Fig. 18, the ratio of the value for $U$ based on $N$ ensembles to the value based on the total number of ensembles available shows that the data is steadily converging towards the maximum sample value indicating that there is a sufficient number of samples ${ }^{38}$ once that number is greater than $\approx 250$. Both the LDV and the PIV datasets are comprised of more than 250 samples, implying that the means have converged in each. The PIV data was examined for evidence of "pixel-locking", which can sometimes occur when there is insufficient particle density in the PIV images. ${ }^{39}$ As shown for a representative plane of PIV data in Fig. 19, evidence of pixel-locking, peaks in the probability mass distribution located at integral pixel displacements, 


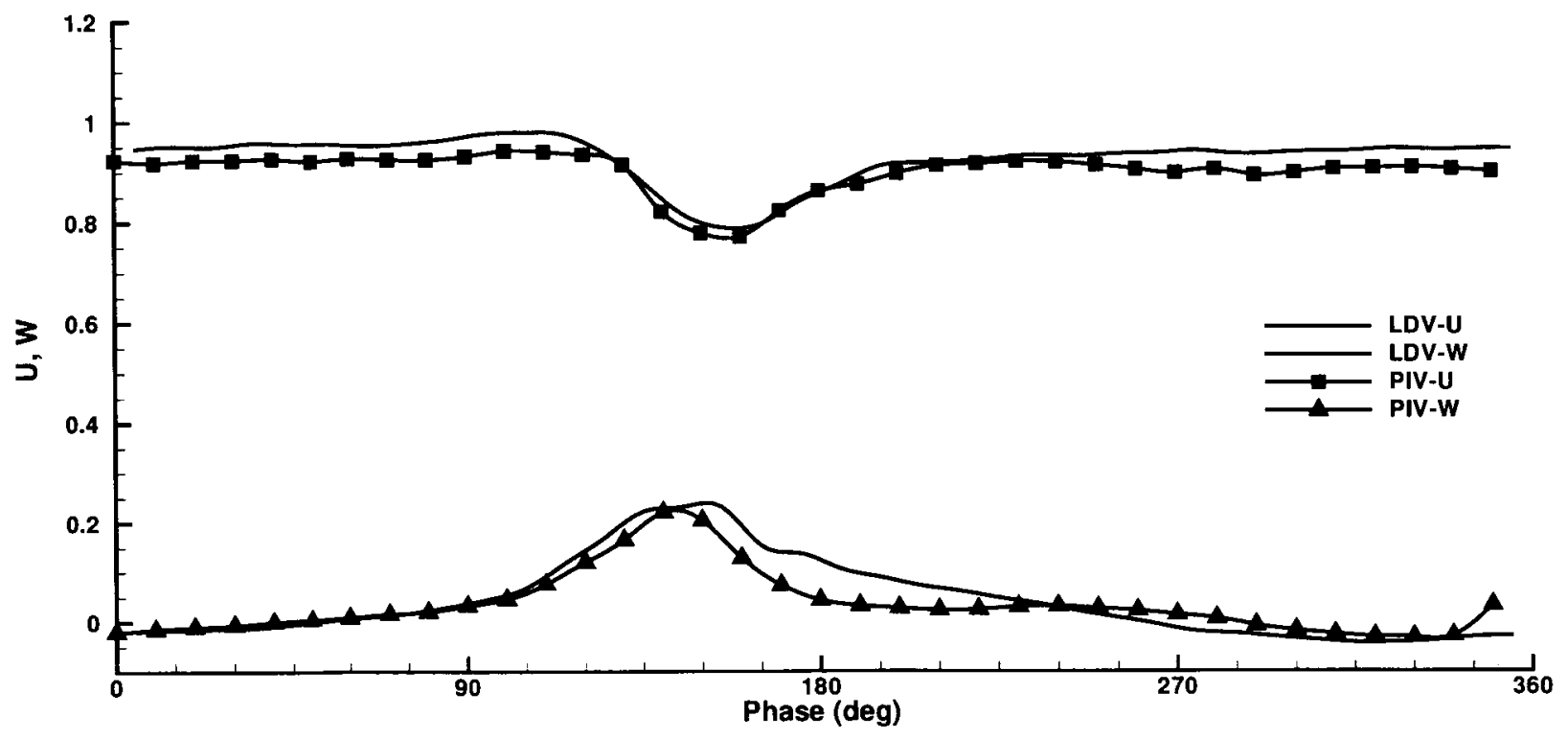

Figure 15. Comparison of the LDV and 2D-PIV data as a function of phase at $(57.1,0.00,14.00)$

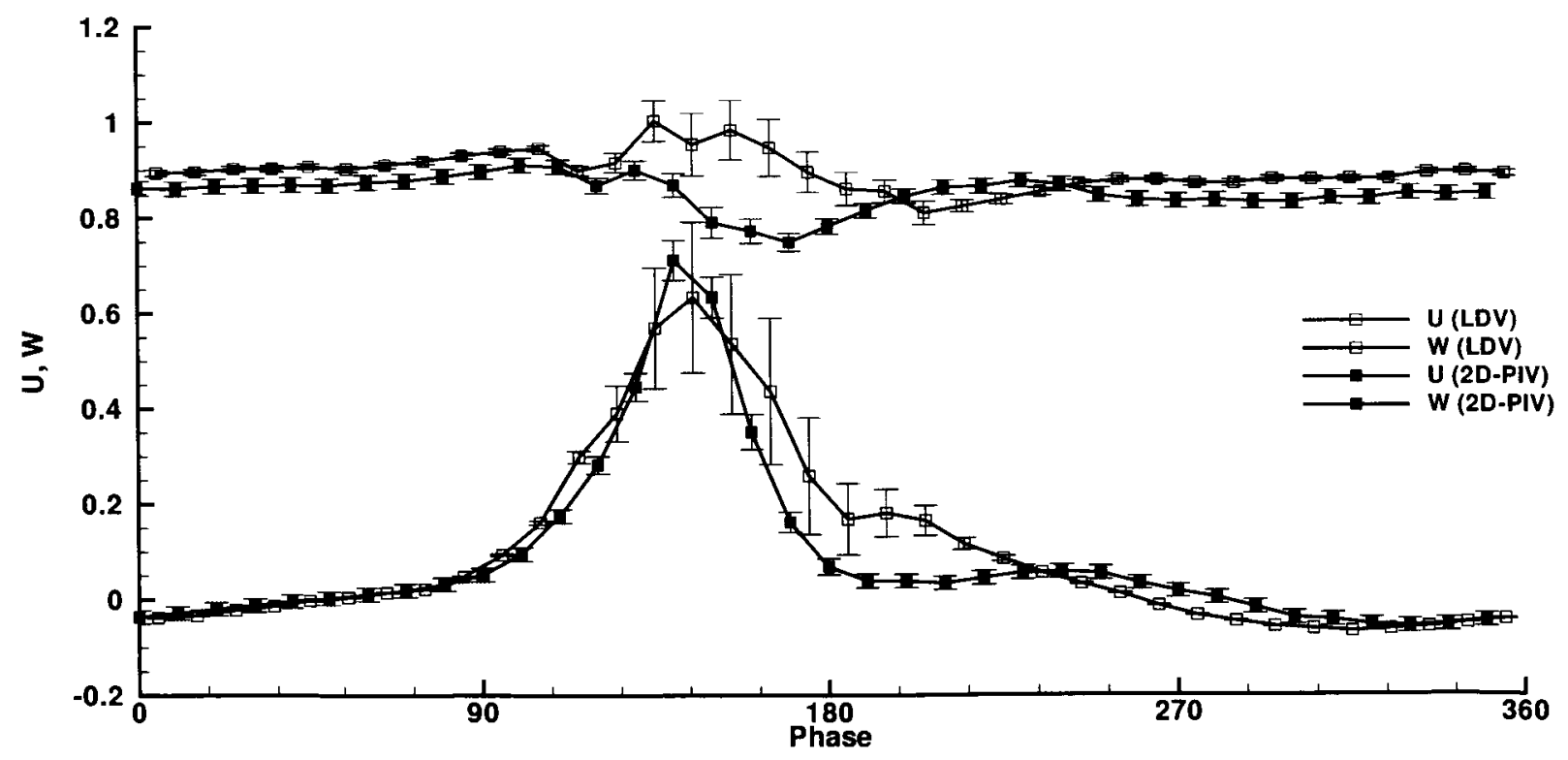

Figure 16. Comparison of the LDV and 2D-PIV data as a function of phase at $(57.1,0.00,10.40)$. Error bars are the complete uncertainty for each measurement. 


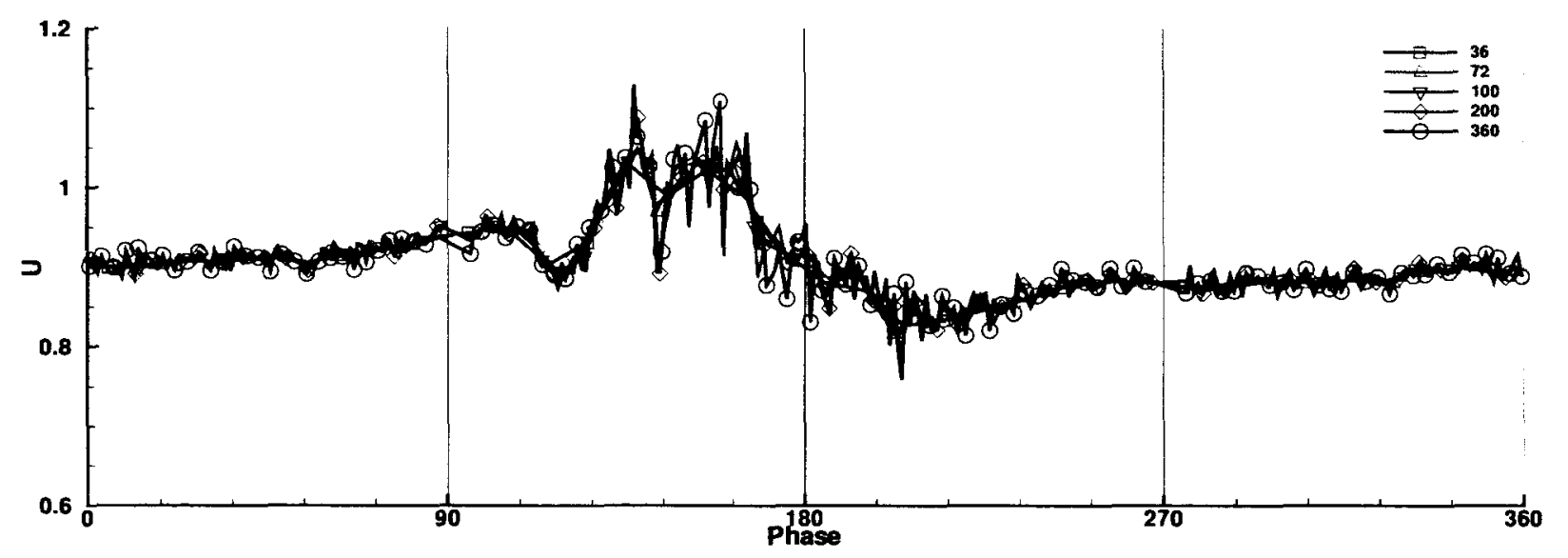

Figure 17. Effect of increasing the number of bins used in phase averaging the LDV data.

was not found. Also the process of removing any cycle-to-cycle variation in the instantaneous PIV velocity fields was examined. The instantaneous PIV velocity fields were low-pass filtered using a LES-style scale decomposition. ${ }^{40}$ These filtered fields were then phase-averaged and a new $U$ velocity signature constructed. As shown in Fig. 20, the filtering does affect the signature during the jet interaction, leaving the signature unaffected elsewhere. This provides an indication that there is some cycle-to-cycle variation. However, there is still an offset with respect to the LDV data that is not uniform and is greater than the offset during the suction cycle.

Finally, the response of the tracer particles themselves was considered. The flow was seeded with $0.86 \mu \mathrm{m}$ polystyrene latex (PSL) particles for the LDV portion of the experiment and seeded with $3.6 \mu \mathrm{m}$ mineral oil droplets during the PIV portion of the experiment. Both particles have a filter-like effect on the measurements of the actual fluid velocity. It is expected that the larger particles used for the PIV will have a larger response time and therefore act more as a low-pass filter to the velocity fluctuations then do the PSL particles. The PIV interrogation method itself, which requires multiple particles to participate in the determination of the velocity as opposed to the single particle required by the coincidence mode LDV, also acts as a filter on the velocity fluctuations that can be measured. ${ }^{41}$ Whether the observed discrepancies in the velocity signatures are solely due to the different particle dynamics in the two techniques is still not clear at this time. It may simply be that the large variance in the flow at this part of the cycle, affects each of the measurements from the two instruments in fundamentally different ways. However, the LDV data is considered to be the better representation of the actual velocity field, since the LDV has a smaller spatial resolution, the measurement is always based on a single micron sized particle, and the effect of the bin size has been demonstrated to be minimal.

\section{Conclusion}

The details of experimental results that support Case 2 of the NASA Langley Research Center Workshop on CFD Validation of Synthetic Jets and Turbulent Separation Control have been documented herein. The specification of the boundary conditions, the in-flow boundary layer profile and turbulence quantities, the geometry of the tunnel and actuator, and the actuator cavity parameters as a function of phase have been documented and presented. A detailed description of the flowfield data acquired by LDV and PIV has been presented, along with a description of the processing and uncertainty of the measurements that are required to arrive at the phase-averaged data. It is hoped that the level of detail documented here and in the data 


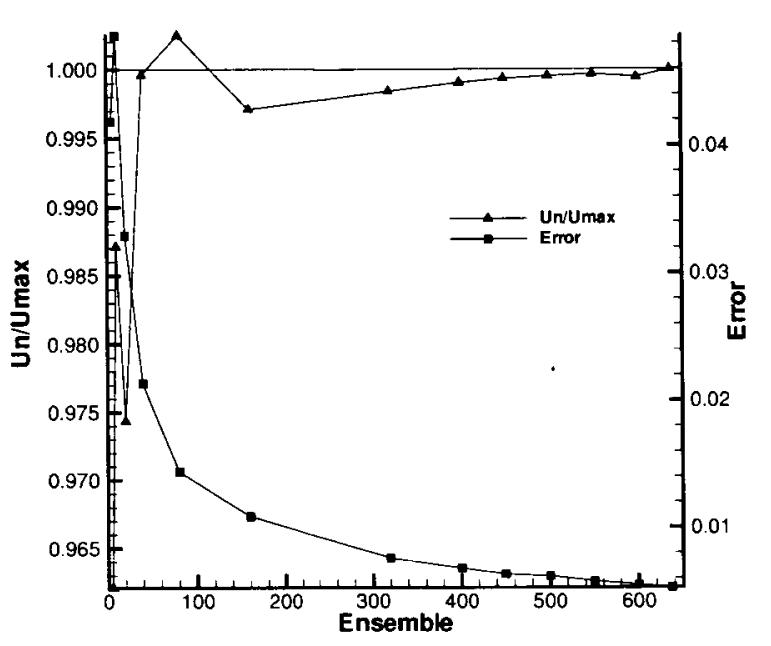

Figure 18. Effect of sample size on the LDV phaseaveraged data.

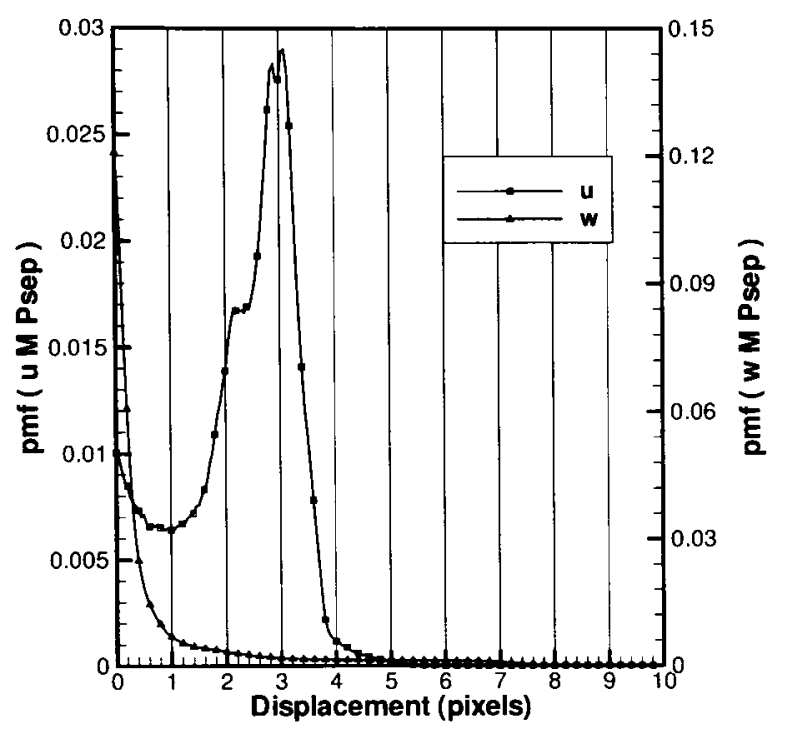

Figure 19. A representative probability mass function (pmf), weighted histogram, for the $u$ and $w$ velocity components as measured by the 2-D PIV. There is no indication of pixel-locking.

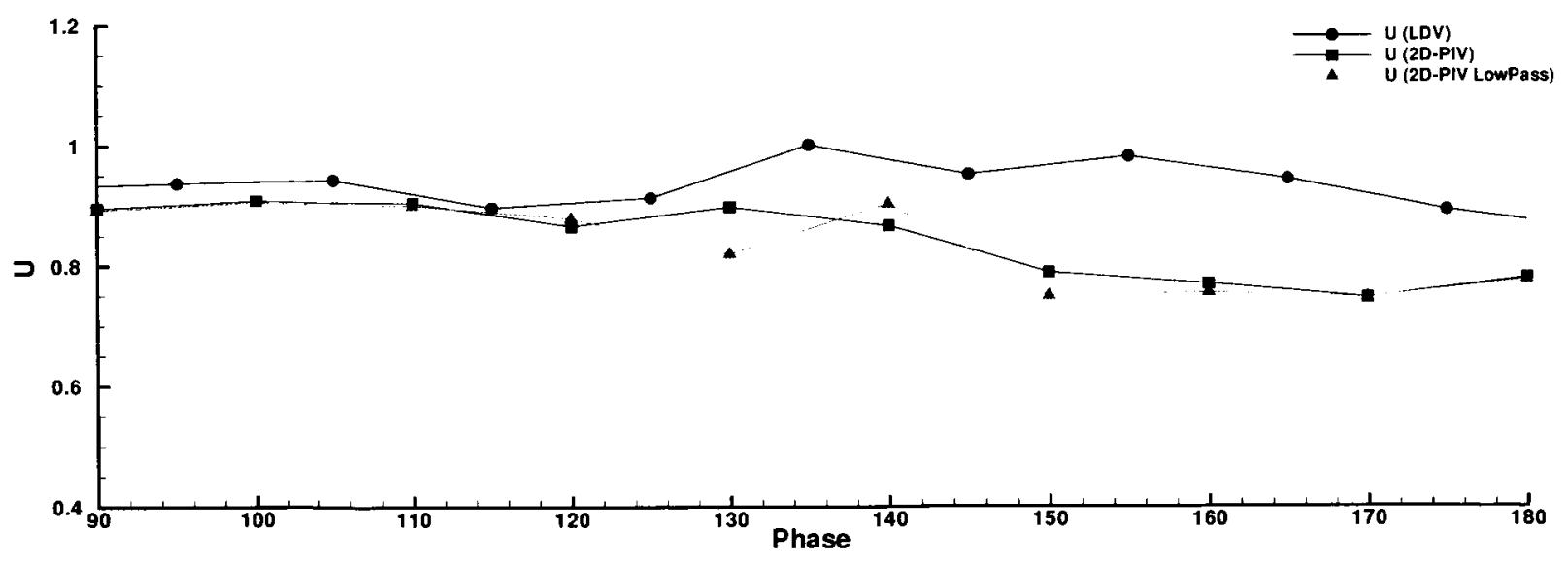

Figure 20. Effect of low pass filtering the instantaneous PIV data before phase averaging. 
publicly available will be adequate to allow this experimental effort to be utilized as a validation database for flow control simulation for years to come.

\section{Appendix}

The experimental data for all three cases of the NASA Langley Research Center Workshop on CFD Validation of Synthetic Jets and Turbulent Separation Control is available via the Web at:

\section{http://cfdval2004.larc.nasa.gov}

\section{Acknowledgments}

The authors would like to thank the following individuals for their support and guidance during the course of this research effort, R. S. Geouge, T. E. Hepner, C. S. Yao, K. B. Paschal, M. J. Walsh, W. L. Sellers, III, and C. L. Rumsey. The actuator utilized here was an ATEAM actuator designed by J. Kiedaisch, H. Nagib, and their associates from IIT. The authors would like to thank Dr. Kiedaisch and Dr. Nagib for providing this actuator to support this research effort. The authors would also like to thank Dr. Flint Thomas and Dr. Patrick Dunn, both of the University of Notre Dame, for conducting the particle sizing experiments.

\section{References}

\footnotetext{
${ }^{1}$ Carpenter, M. H., Singer, B. A., Yamaleev, N., Vasta, V. N., Viken, S. A., and Atkins, H. L., "The Current Status of Unsteady CFD Approaches for Aerodynamic Flow Control," AIAA Paper 2002-3346, 2002.

${ }^{2}$ Rumsey, C., Gatski, T., Sellers, W. L., Vatsa, V., and Viken, S., "Summary of the 2004 CFD Validation Workshop on Synthetic Jets and Turbulent Separation Control," AIAA Paper 2004-2217, 2004.

${ }^{3}$ Smith, B. L. and Glezer, A., "The Formation and Evolution of Synthetic Jets," Physics of Fluids, Vol. 10, No. 9, 1998, pp. 2281-2297.

${ }^{4}$ Glezer, A. and Amitay, M., "Synthetic Jets," Annual Review of Fluid Mechanics, No. 34, Annual Reviews, Palo Alto, California, 2002, pp. $503-529$.

${ }^{5}$ Amitay, M., Honohan, A., , Trautman, M., and Glezer, A., "Modification of the Aerodynamic Characteristics of Bluff Bodies Using Fluidic Actuators," AIAA Paper 97-2004, 1997.

${ }^{6}$ Béra, J. C. and Sunyach, M., "Control of Boundary Layer Separation by Jet Oscillation," AIAA Paper 98-2373, 1998.

${ }^{7}$ Wood, N. J., Sadri, A. M., and Crook, A., "Control of Turbulent Flow Separation by Synthetic Jets," AIAA Paper 2000-4331, 2002.

${ }^{8}$ Amitay, M., Kibens, V., Parekh, D., and Glezer, A., "The Dynamics of Flow Reattachment over a thick Airfoil Controlled by Synthetic Jet Actuators," AIAA Paper 99-1001, 1999.

${ }^{9}$ Washburn, A. E., "NASA Micro-Aero-Adaptive-Control," SPIE Paper 4332-39, SPIE 8th Annual International Symposium on Smart Structures and Materials, March, 2001.

${ }^{10}$ Chatlynne, E., Rumigny, N., Amitay, M., and Glezer, A., "Virtual Aero-shaping of a Clark-Y Airfoil Using Synthetic Jet Actuators," AIAA Paper 2001-0732, 2001.

${ }^{11}$ Amitay, M., Smith, D. R., Kibens, V., Parekh, D. E., and Glezer, A., "Aerodynamic Flow Control over an Unconventional Airfoil Using Synthetic Jet Actuators," AIAA Journal, Vol. 39, No. 3, 2001, pp. 361-370.

${ }^{12}$ McCormick, D. C., "Boundary Layer Separation Control with Directed Synthetic Jets," AIAA Paper 2000-0519, 2000.

${ }^{13}$ Jenkins, L., Althoff Gorton, S., and Anders, A., "Flow Control Device Evaluation for an Internal Flow with an Adverse Pressure Gradient," AIAA Paper 2002-0266, 2002.

${ }^{14}$ Yao, C. S., Chen, F., and Harris, J., "Synthetic Jet Flow Field Database for CFD Validation," AIAA Paper 2004-2218, 2004.

${ }^{15}$ Greenblatt, D., Paschal, K., Schaeffler, N. W., Washburn, A., and Yao, C. S., "Separation Control over a Wall-Mounted Hump: A CFD Validation Test Case," AIAA Paper 2004-2220, 2004.

${ }^{16}$ Schaeffler, N. W., "The Interaction of a Synthetic Jet and a Turbulent Boundary Layer," AIAA Paper 2003-0643, 2003.

${ }^{17}$ Kelso, R. M., Lim, T. T., and Perry, A. E., "An experimental study of round jets in cross-flow," Journal of Fluid Mechanics, Vol. 306, 1998, pp. 111-144.

${ }^{18}$ Kelso, R. M., Lim, T. T., and Perry, A. E., "New experimental observations of vortical motions in traverse jets," Physics of Fluids, Vol. 10, No. 9, 1998, pp. 2427-2429.
} 
${ }^{19}$ Fric, T. F. and Roshko, A., "Vortical structure in the wake of a transverse jet," Journal of Fluid Mechanics, Vol. 279, 1994, pp. 1-47.

${ }^{20}$ Eroglu, A. and Breidenthal, R. E., "Structure, Penetration, and Mixing of Pulsed Jets in Crossflow," AIAA Journal, Vol. 39, No. 3, 2001, pp. 417-423.

${ }^{21}$ Johari, H., Pacheco-Tougas, M., and Hermanson, J. C., "Penetration and Mixing of Fully Modulated Turbulent Jets in Crossflow," AIA A Journal, Vol. 37, No. 7, 1999, pp. 842-850.

${ }^{22}$ Chang, Y. K. and Vakili, A. D., "Dynamics of Vortex Rings in Crossflow," Physics of Fluids, Vol. 7, No. 7, 1995, pp. 15831597.

${ }^{23}$ Smith, D. R., "Interaction of a Synthetic Jet with a Crossflow Boundary Layer," AIAA Journal, Vol. 40, No. 11, 2002, pp. $2277-2288$.

${ }^{24}$ Gordon, M. and Soria, J., "PIV measuements of a zero-mass-flux jet in crossflow," Experiments In Fluids, Vol. 33, 2002, pp. 863-872.

${ }^{25}$ Zaman, K. B. M. Q. and Milanovic, I. M., "Synthetic Jets in Cross-Flow. Part 1: Round Jet," AIAA Paper 2003-3714, 2003. ${ }^{26}$ Mittal, R., Rampunggoon, P., and Udaykumar, H. S., "Interaction of a Synthetic Jet with a Flat Plate Boundary Layer," AIAA Paper 2001-2773, 2001.

${ }^{27}$ Mittal, R. and Rampunggoon, P., "On the virtual aeroshaping effect of synthetic jets," Physics of Fluids, Vol. 14, No. 4, 2002, pp. 1533-1536.

${ }^{28}$ Albrecht, H. E., Borys, M., Damaschke, N., and Tropea, C., Laser Doppler and Phase Doppler Measurement Techniques, Springer-Verlang, 2003

${ }^{29}$ Durst, F., Melling, A., and Whithall, J. H., Principles and Practice of Laser-Doppler Anemometry, Academic Press, 2nd ed., 1981.

${ }^{30}$ Willert, C. E. and Gharib, M., "Digital particle image velocimetry," Experiments In Fluids, Vol. 10, 1991 , pp. $181-193$.

${ }^{31}$ Westerweel, J., Digital Particle Image Velocimetry - Theory and Application, Ph.D. thesis, Delft University of 'Technology, The Netherlands, 1993.

${ }^{32}$ Raffel, M., Wilert, C., and Kompenhans, J., Particle Image Velocimetry: A Practical Guide, Springer-Verlang, 1998.

${ }^{33}$ Nichols, C. E., "Preparation of Polystyrene Microspheres for Laser Velocimetry in Wind Tunnels," NASA TM-89163, 1987.

${ }^{34}$ Klebanoff, P. S., "Characteristics of Turbulence in a Boundary Layer with Zero Pressure Gradient," NACA TR-1247, 1953.

${ }^{35}$ Coleman, H. W. and Steele, W. G., Experimentation and Uncertainty Analysis for Engineers, John Wiley and Sons, 1999.

${ }^{36}$ Benedict, L. H. and Gould, R. D., "Towards better uncertainty estimates for turbulent statistics," Experiments in Fluids, 1996 , pp. $129-136$.

${ }^{37} \mathrm{Uzol}$, O. and Camci, C., "The effect of sample size, turbulence intensity and the velocity field on the experimental accuracy of ensemble averaged PIV measurements," 4th International Symposium on Particle Image Velocimetry, September 2001.

${ }^{38}$ Wernert, P. and Favier, D., "Considerations about the phase averaging method with application to ELDV and PIV measurements over pitching airfoils," Experiments In Fluids, Vol. 27, 1999, pp. 473-483.

${ }^{39}$ Christensen, K. T., "The Influence of Peak-locking Errors on Turbulence Statistics Computed from PIV Ensembles," Experiments in Fluids, Vol. 36, 2004, pp. 484-497.

${ }^{40}$ Adrian, R. J., Christensen, K. T., and Liu, Z.-C., "Analysis and interpretation of instantaneous turbulent velocity fields," Experients in Fluids, Vol. 29, 2000, pp. 275-290.

${ }^{41}$ Lecordier, B., Demare, D., Vervisch, L. M. J., Réveillon, J., and Trinité, M., "Estimation of the accuracy of PIV treatments for turbulent flow studies by direct numerical simulation of multi-phase flow," Measurment Science and Technology, Vol. 12, 2001, pp. 1382-1391. 